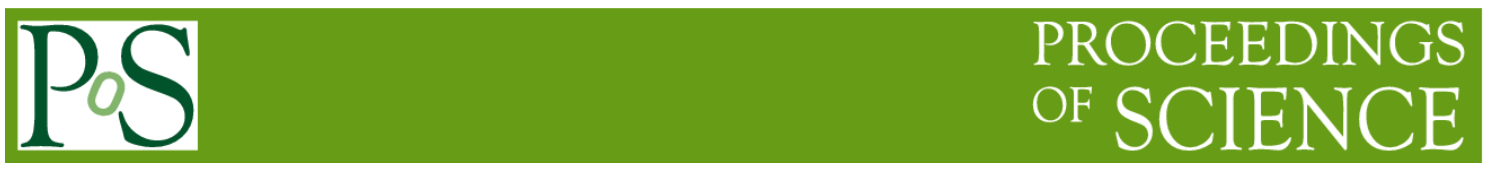

\title{
Tracking Cosmic Rays by CRAYFIS (Cosmic Rays Found in Smartphones) Global Detector
}

\section{Rajiv Kumar ${ }^{1}$}

Government Pench Valley PG college

Parasia Distt. CHHINDWARA M.P., INDIA

E-mail: captainrajivelive.com

ABSTRACT:The purpose of this research paper is to develop efficient site arrays of using CRAYFIS(Cosmic Rays Found in Smartphones) which gives real time-radiation map on temporal and spatial scales.It is suggested how a map can be prepared in terms of exact azimuth,zenith and altitude angles so that the direction of the cosmic rays could be estimated.

KEY WORDS: CRAYFIS,Zenith,Azimuth angle.

The 34th International Cosmic Ray Conference

30 July- 6 August, 2015

The Hague, The Netherlands

\footnotetext{
${ }^{1}$ Rajiv Kumar
} 
1. Introduction

When cosmic rays strike the atmosphere, they produce a huge number of lower energy particles in an extensive air shower, which can stretch to many kilometers across. The very high-energy cosmic rays produce the largest showers, but unfortunately there are very rare. Only a detector with large surface area can observe these mysterious particles.

The CRAFIS(Cosmic Rays Found In Smartphones) detector array preliminary applications for Android and iOS platform are available for testing [1]. Smartphones can detect ionizing radiation[2,3].

UHECRs(Ultra-high Energy Cosmic Rays) produce extensive air showers, Which can be detected via the particle flux on the ground, the fluorescence in the air, or the radio and acoustic signatures. A series of dedicated detectors [4-6] have detected cosmic rays at successively higher energies, culminating in observation upto $3.10^{20} \mathrm{eV}$. The flux of particles drops precipitously above $10^{18} \mathrm{GeV}$, due to the suppression via interaction with the cosmic microwave background[7,8],making observation of these particles challenging .In this paper, we demonstrate that dense network of such devices has power sufficient to detect air showers and also the trajectory of the highest energy cosmic rays.

The source of UHECRs those with $10^{18} \mathrm{eV}$, remains a puzzle even many decades after their discovery, as does the mechanism behind their acceleration. Their high energy leaves them less susceptible to bending by magnetic fields between their source and the Earth making them excellent probes of the cosmic acceleraters which produce them [9,10].My effort would be to point these sources.

\section{Detection}

Cosmic rays induce air showers which contain an enormous number of particles Fig 1 shows the energy spectrum, and spatial distribution at sea level of photons, electrons, and muons in showers as simulated by CORSIKA [10] program with the QGS-II[11] model of hadronic interactions.

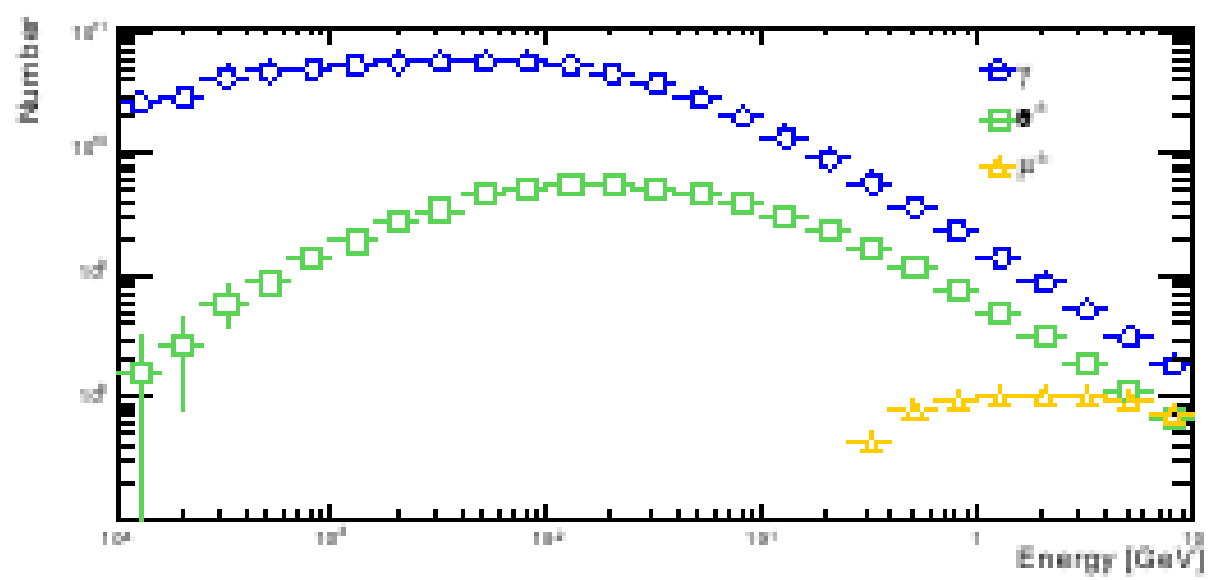




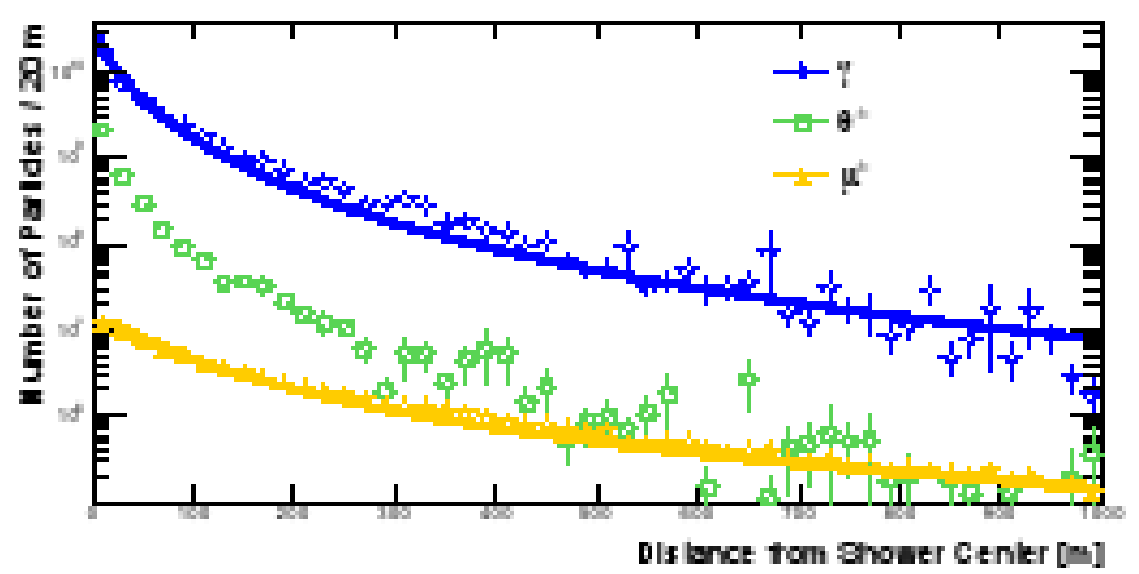

Fig 1 Energy Spectrum (a) and distance from shower axis(b) of photons, electrons and muons at ground level for simulated air showers initiated by protons with energy $10^{19}$. $10^{20} \mathrm{eV}$.

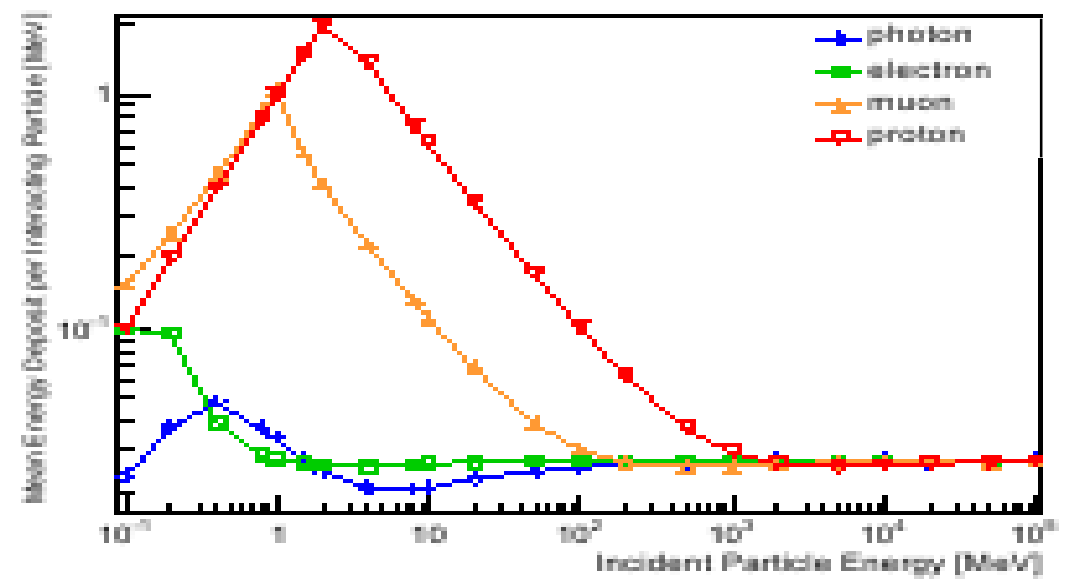

(A)

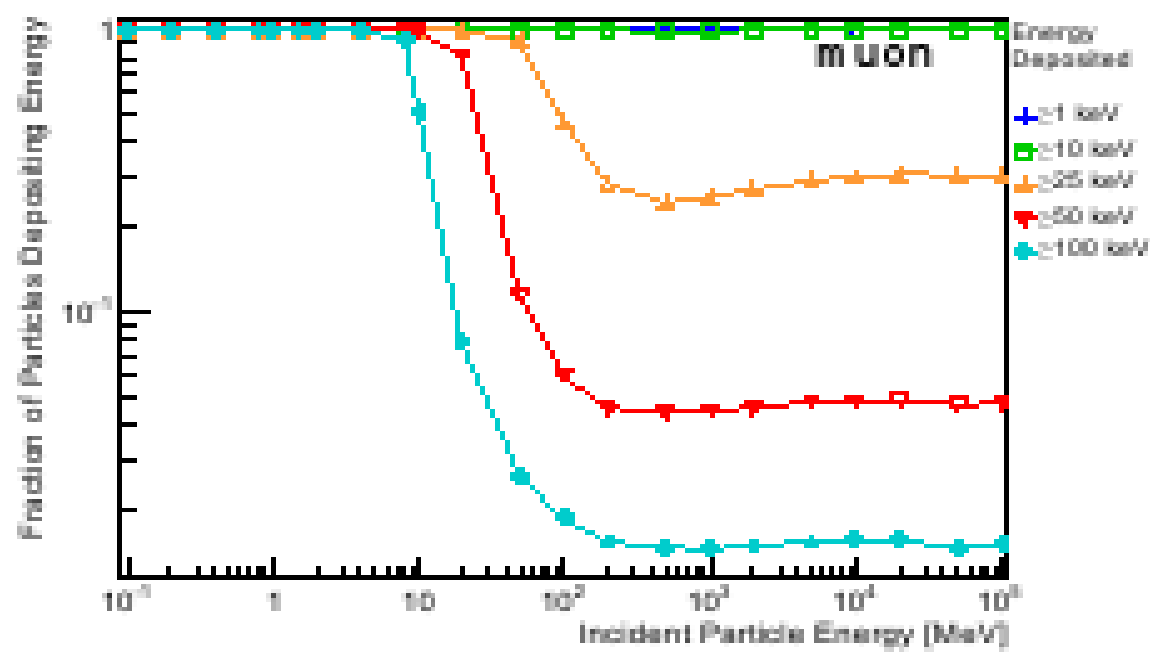


(B)

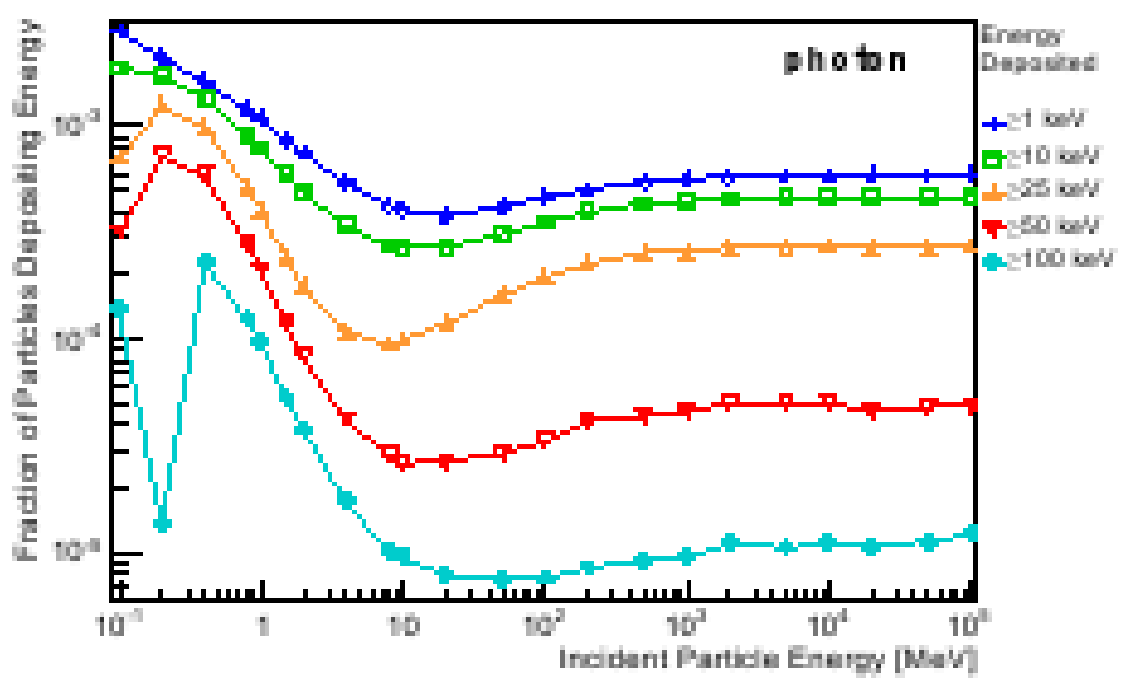

(C)

Fig. 2: Studies of energy deposition by photons, electrons and muons 50 micrometer thick Si in GEANT simulations.(A)Shows the mean energy deposition per interacting particle.(B) (C) Shows the fraction of incident photons (muons) which deposit energy above a set of thresholds.

The focus is on photons, which have high densities in the shower, and muons, which have excellent penetrating power and high detection efficiency. Electrons are also numerous and have high efficiency, but many be blocked by buildings, phone cases or camera lenses. Heavier hadronic particles are much less common.

The sensitive element in a smartphone is the camera, a CMOS device in which silicon photodiode pixels are designed to absorb visible photons and convert them to current which is collected and read out. While these devices are designed to have reasonable quantum efficiency for visible light, the same principle allows the sensor to detect higher-energy photons[12] as well. In the case of muons, the photodiode is functionally equivalent to silicon-based trackers now common in particle physics experiments, such that the charged particle will leave electron-hole pairs along the path.

The energy deposition of photons in silicon indicates that the camera sensor has efficiency over the energy range of interest (Fig. 2) GEANT-based simulation[13]. 
The phone processor runs an application which functions as the trigger and data acquisition system. To obtain the largest possible integrated exposure time, the first -level trigger capture video at 15-30 frames per second, depending on the frameprocessing speed of the device. Frame which contain any above threshold pixels are stored and passed to the second stage which examines the stored frames, saving only the pixels above a second, lower threshold. All qualifying pixels, typically a few per frame, are stored as a sparse array in a buffer on the phone, along with their arrival time and geo-location of the phone. When a wi-fi connection is available, the collected pixels are uploaded to a central server for offline shower reconstruction.

The application runs when the phone is not in active use. It launches itself when it detects a power source, and quits when the power is disconnected. No additional light schielding such as tape, of the camera is required other than placing the phone face-up (camera-down).

\section{Finding Direction of UHECR}

Considering that phenomenon of air showers are isotropic The projection of the air shower would be approximate conic section on the earth surface and the direction of the UHECR is the axis of this cone so if we could get the projection of air shower the direction of incident UHECR could be estimated and hence the source from which it is coming could be located since the high energy cosmic rays leaves them less susceptible to bending by magnetic field between their source and the Earth, making them excellent probes of the cosmic accelerators which produce them $[14,15]$.

In order to record the projection CRAFIS smart phones are located in close regular sites once an evenly distributed sites are organized the trace of the projection could be recorded

For this purpose set of data could be obtained in real time in terms of azimuth and altitude angles at various zenith

\section{Suggested Sites for CRAYFIS smart phones}

smart phones which are not in use can be used to prepare a net of floats which has tiny solar pannel to charge them connected through a Wi-Fi network this would form an excellent detector at sea level.

At land the network of CRAYFIS volunteer could be used as to detect the air shower projection. 
Acknowledgement: The Author is thankful to Daniel Whiteson, Michael Mulhearn ,Chase Shimmin Kyle Brodie and Dustin Burns and entire CRAYFIS team For this novel idea of CRAYFIS and many Who supported

\section{References}

. URL http://crafis.ps.uci.edu/.

[1] J. J. Cogliati, K. W. Derr, and J. Wharton (2014),1401.0766.

[2] A. R. Smith, R. J. McDonald, D. C.Hurley, S. E. Holand, D.E. Groom, W. E. Brown, D. K. Gilmore, R. J.Stover, and M. Wei, in sensors and Camera Systems for scientific, Industrial, Digital Photography Applications III,vol. 4669 of Society of Photo- Optical Instrumentation Engineers (SPIE) Conference Series, pp.172-183.

[3] R. Abbasi et al.(High Resolution Fly’s Eye Colaboration), Phys, Rev.Lett. 92, 151101 (2004), astroph/0208243.

[4] M. Takeda, N. Hayashida, K. Honda, N. Inoue, K. Kodata, et al, Phys. Rev.Ltt.81,1163(1998),astroph/9807193.

[5] J. Abraham et al. (Pierre Auger Collaboration),Phys.Rev.Lett. 101,061101(2008),0806.4302.

[6] K. Geisen, Phys.Rev.Lett. 16,748(1966).

[7] G. Zatsepin and V. Kuzmin, JETP Lett. 4,78(1966).

[8] J. Abraham et ai.(Pierre Auger collaboration), Astropart. Phys.29, 188(2008), 0712.2843.

[9] D. Herk, G. Schatz, T. Thouw, J. Knapp, and J. Capdevielle (1998).

[10] S. Ostapchenko, Phys. Rev. D83, 014018 (2011), 1010.1869.

[11] J. J. Cogliati, K. W. Derr, and J. Wharton (2014),1401.0766.

[12] S. Agostinelli et al. (GEANT4),Nucl.Instrum.Meth.A506,250(2003),1307.5059.

[13] J. Abraham et al. (Pierre Auger Collaboration), Astropart.Phys. 29, 188 (2008),0712.2843

[14] J. Abraham et al. (Pierre Auger Collaboration), Science 318,938 (2007), 0711,2256. 
Title (or short title)

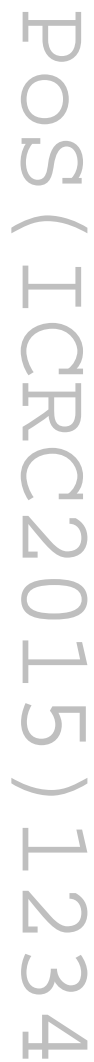

